\title{
Anatomical considerations during Laparoscopic excision of a splenic cyst: Case Report
}

Jayamaha JHPWB ${ }^{1}$, Sopan $\mathrm{S}^{1}$, Galketiya $\mathrm{KB}^{1}$

${ }^{I}$ Teaching Hospital Peradeniya, Sri lanka

\section{Introduction}

Splenic cysts are an uncommon disease entity of the spleen. Most of the patients are asymptomatic and are diagnosed incidentally in abdominal ultra sound and CT scans that are performed for other reasons. Some present with vague abdominal pain with or without mass.

Performing excision by laparoscopy reduces morbidity. To perform laparoscopic excision anatomy of the spleen provides the key for positioning of patient, port placement and subsequent procedure.

We present a patient who underwent a laparoscopic excision of a splenic cyst.

\section{Case Report}

A 58 year old female presented with a vague aching type persistent epigastric and left hypochondrial pain for two months. There was no history of abdominal trauma. General and abdominal examinations were normal. Ultrasound scan revealed a unilocular thin walled encapsulated cyst in the lower pole of spleen measuring $7.9 \mathrm{~cm} \mathrm{x}$ $7.1 \mathrm{~cm} \times 5.9 \mathrm{~cm}$ suggestive of a benign cyst. Contrast $\mathrm{CT}$ abdomen confirmed an encapsulated unilocular thin walled cyst at lower pole of the spleen.
Laparoscopic splenic cyst excision was planned after obtaining informed consent. As the spleen and cyst occupies the left hypochondrium patient was placed on right lateral decubitus position and head up tilt to let other organs fall away from field of dissection. With stomach's close relationship with the spleen decompression of stomach with a nasogastric tube facilitates dissection. Surgery was performed using three ports. After inserting the camera port related anatomy was appreciated. Stomach, spleen, transeverse colon, splenic flexure, gastro-splenic ligament, gastro-colic ligament and spleno-colic ligament were identified. As the cyst was large it was initially decompressed by aspiration. Excision of the cyst with a thin rim of splenic tissue was done using ultra-sonic dissector and bipolar diathermy. Hilar area with splenic vasculature was preserved. The specimen was retrieved within a retrieval bag through enlarged $10 \mathrm{~mm}$ port site. The surgery was completed in 45 minutes without any measurable blood loss.

Patient was commenced on oral feeding on same day and mobilized out of bed. Pain was managed with oral analgesics and Diclofenac sodium suppositories. 
The patient was discharged on the following day. The histology revealed a pseudocyst with chronic granulomatous inflammation suggestive of

Tuberculosis. She was referred to chest clinic where Mantoux test was strongly positive and was started on anti-TB therapy.

\section{Discussion}

According to Fowler's classification, the splenic cysts are primary or true and secondary or false cysts depending on the presence or absence of true epithelial lining. Primary cysts can be congenital, traumatic, inflammatory, neoplastic or parasitic and secondary cysts can be traumatic, inflammatory or degenerative ${ }^{[1]}$.

Most of the splenic cysts are asymptomatic. Patients can present with vague left hypochondrial pain, left side shoulder tip pain and postprandial abdominal fullness. They also can present with complications of cysts which includes trauma leading to rupture and haemorrhage and infection.

Contrast enhanced CT is the investigation of choice for splenic cysts specially in differentiating nonparasitic cysts from parasitic cysts ${ }^{[2]}$. The key to interpret the CT is understanding crosssectional anatomy of abdomen. Non parasitic cysts are unilocular densely cystic and thin walled whereas parasitic cysts are multilocular with daughter cysts within the main cysts ${ }^{[3]}$.
Asymptomatic cysts less than $4 \mathrm{~cm}$ can be managed conservatively. Symptomatic cysts and asymptomatic cysts more than $4 \mathrm{~cm}$ warrant intervention $^{[4]}$.

Treatment options include percutaneous drainage, laparoscopic total cyst excision, laparoscopic partial splenectomy, laparoscopic partial cyst excision with marsupialization and hand assisted laparoscopic splenectomy. The laparoscopic approach is associated with lower morbidity when compared to open surgery ${ }^{[1]}$. Though laparoscopic total cyst excision or partial splenectomy removes the cyst completely and reduces the risk of recurrence, it is more time consuming and has higher bleeding risk compared to partial cyst excision with marsupialization. However with perception of anatomy of spleen and related organs the procedure was completed in forty five minutes without any measurable blood loss. The recurrence rates are $64 \%$ and $78 \%$ respectively ${ }^{[5]}$. Measures such as Argon laser coagulation of splenic surface and omental packing do not completely eliminate the risk of recurrence.

The patient discussed underwent a complete cyst excision with a thin rim of splenic tissue. The histopatholgy revealed a chronic granulomatous pathology suggestive of Tuberculosis, Melioidosis or parasitic infection. Patient was referred to a chest physician and was started on anti-TB therapy as mantoux test was strongly positive. 


\section{Conclusion}

This case illustrates that appreciation of anatomy is the basis of making a surgical diagnosis including interpretation of CT images. Complete excision of a splenic cyst can be performed by laparoscopically and detailed appreciation of anatomy provides the key.

Key words: Laparoscopy, Splenic cyst

\section{References}

1. Dilip Dan.Laparoscopic Management of a Massive Splenic cyst.Asian Journal of Surgery.2010Jan;33(2)103-6).
2. Williams RJL. Splenic cysts: changes in diagnosis, treatment and etiological concepts. Ann R Coll Surg Engl 1993;75:87-9

3. Adas G, Karatepe O, Altiok M, et al. Diagnostic problems with parasitic and non-parasitic splenic cyst. BMC Surg 2009;9:9.

4. Lasonof JE, Richman BN, Jones JW. Hand assisted laparoscopic surgery of splenic cysts. J Laparoendosc Adv Surg Tech A 2003;13: 339-40. 7

5. Czauderna $P$, Vajda $P$, Schaarschmidt K, et al. Nonparasitic splenic cyst in children: a multicentric study. Eur J Pediatr Surg 2006;16:415- 